\title{
On a New Optimization Method With Constraints
}

\author{
Bouchta RHANIZAR
}

Correspondence: Departement of Mathematics, Ecole Normale Supérieure, University Mohammed V of Rabat, Morocco

Received: July 2, 2020 Accepted: August 14, 2020 Online Published: August 24, 2020

doi:10.5539/jmr.v12n5p27

URL: https://doi.org/10.5539/jmr.v12n5p27

\section{Abstract}

We consider the constrained optimization problem defined by:

$$
f\left(x^{*}\right)=\min _{x \in X} f(x)
$$

where the function $f: \mathbb{R}^{n} \longrightarrow \mathbb{R}$ is convex on a closed convex set $X$.

In this work, we will give a new method to solve problem (1) without bringing it back to an unconstrained problem. We study the convergence of this new method and give numerical examples.

Keywords: nonlinear optimization

\section{Introduction}

In many fields of science we need to solve some non-linear optimization problems. Several authors have studied the resolution of non-linear optimization problems, for example (Dennis \& Schnabel, 1983; Ortega \& Rheinboldt, 1970; Gilbert, 2009) and (Rhanizar, 2002). Our goal is to propose a new constrained optimization method without turning in to an unconstrained optimization problem. Among the methods used to solve the problem (1) by transforming it to an unconstrained problem, we can cite the projection methods defined by:

$$
x_{k+1}=P_{X}\left(x_{k}-\alpha_{k} \nabla f\left(x_{k}\right)\right)
$$

with

$$
\left\|x-P_{X}(x)\right\|=\min _{y \in X}\|x-y\|
$$

Calculating the projection on $X$ can sometimes be more difficult than the initial problem. In this paper, we propose a new method to solve the problem (1) based on defining a descent direction and a step while staying in the convex domain $X$.

In order to present conveniently our results, let us introduce the setting used throughout this paper. We use the following notations:

$\|x\|=\left(\sum_{i=1}^{n} x_{i}^{2}\right)^{\frac{1}{2}}$, the Euclidean norm.

( , ) the corresponding inner product.

$\nabla f(x)=\left(\frac{\partial f}{\partial x_{i}}(x)\right)_{1 \leq i \leq n}$ : the gradient of the function $f$.

$\nabla^{2} f(x)=\left(\frac{\partial^{2} f}{\partial^{2} x_{i} x_{j}}(x)\right)_{\substack{1 \leq i \leq n \\ 1 \leq j \leq n}}:$ the Hessian matrix.

This work is organized as follows:

The introduction is in section 1. In section 2, we give how to choose the direction of descent and a new algorithm that solves problem (1). Section 3 is devoted to results of convergence of the new method. In section 4, we study the speed of convergence. In section 5, some numerical examples are elaborated. Finally, the conclusions form section 6.

\section{Searching for a Direction of Descent}

At step k, we will define a direction $d_{k}$ which checks $\left(\nabla f\left(x_{k}\right), d_{k}\right) \leq 0$.

We look for $x_{k+1}$ of the form $x_{k+1}=x_{k}+\alpha_{k} d_{k}$ such that:

1). $x_{k+1} \in X$, this condition for not using the projection on $X$ 
2). $\alpha_{k}>0$

3). $f\left(x_{k+1}\right)-f\left(x_{k}\right) \leq 0$

On the other hand, we have $f\left(x_{k+1}\right)-f\left(x_{k}\right) \approx \nabla f\left(x_{k}\right)\left(x_{k+1}-x_{k}\right)=\nabla f\left(x_{k}\right) \alpha_{k} d_{k}$

To get 1), just take $0<\alpha_{k}<1$ and $d_{k}=y_{k}-x_{k}$ with $y_{k} \in X$

For 3$)$ to be verified, it is enough $\nabla f\left(x_{k}\right)\left(y_{k}-x_{k}\right) \leq 0$ which gives $\nabla f\left(x_{k}\right) y_{k} \leq \nabla f\left(x_{k}\right) x_{k}$

It is thus finally enough to choose $y_{k}$ such as:

$$
\left(\nabla f\left(x_{k}\right), y_{k}\right)=\min _{y \in X}\left(\nabla f\left(x_{k}\right), y\right)
$$

Condition 3) is not sufficient for convergence (Rondepierre, A. (2017)). This is why we are going to impose that:

$$
f\left(x_{k}+\alpha_{k} d_{k}\right)-f\left(x_{k}\right) \leq \frac{1}{2} \alpha_{k}\left(\nabla f\left(x_{k}\right), d_{k}\right)
$$

For this direction $d_{k}=y_{k}-x_{k}$, two cases arise:

First case: If $d_{k}=0$, we have $y_{k}=x_{k}$ so:

$$
\left(\nabla f\left(x_{k}\right), x_{k}\right) \leq\left(\nabla f\left(x_{k}\right), y\right) \forall y \in X
$$

and thereafter:

$$
\left(\nabla f\left(x_{k}\right), x_{k}-y\right) \leq 0 \forall y \in X
$$

therefore $x_{k}$ is the solution to problem (1) according to the optimization conditions.

Second case: If $d_{k} \neq 0$

we have $\left(\nabla f\left(x_{k}\right), y_{k}\right) \leq\left(\nabla f\left(x_{k}\right), x_{k}\right)$ therefore $\left(\nabla f\left(x_{k}\right), d_{k}\right) \leq 0$

which gives $d_{k}$ is a direction of descent.

So we have the following algorithm:

\section{Algorithm}

1. Choose $\left.x_{0} \in X, \epsilon \in\right] 0,1\left[, \alpha_{0} \in\right] 0,1[$ and $k:=0$

2. At step $k$

\subsection{Compute $\nabla f\left(x_{k}\right)$}

2.2. Compute $y_{k} \in X$ by: (2)

2.3. Set $d_{k}=y_{k}-x_{k}$

3. If $\left|\left(\nabla f\left(x_{k}\right), d_{k}\right)\right| \leq \epsilon$, then:

3.1. Set $x^{*}=x_{k}$

3.2. Stop

end If

4. Compute $x_{k}+\alpha_{k} d_{k}$

5. If $f\left(x_{k}+\alpha_{k} d_{k}\right)-f\left(x_{k}\right) \leq \frac{1}{2} \alpha_{k}\left(\nabla f\left(x_{k}\right), d_{k}\right)$

5.1. $x_{k+1}=x_{k}+\alpha_{k} d_{k}$

5.2. Set $k:=k+1$ and go to 2 .

end If

6. $\alpha_{k}=\frac{1}{2} \alpha_{k}$ and go to 4 . 


\section{Convergence Study}

The possible choice of $\alpha_{k}$ and the convergence of the sequence $\left(x_{k}\right)_{k}$ to the solution $x^{*}$ are given by the following theorem:

Theorem 1 Let $f$ be of class $C^{2}$ on $X$ convex bounded, and suppose that there exists $m>0$ and $M>0$, such that:

$$
m\|y\|^{2} \leq\left(\nabla^{2} f(x) y, y\right) \leq M\|y\|^{2} \quad \forall x, y \in X
$$

Then:

1. $x_{k} \in X \quad \forall k \geq 0$

2. Condition $f\left(x_{k}+\alpha_{k} d_{k}\right)-f\left(x_{k}\right) \leq \frac{1}{2} \alpha_{k}\left(\nabla f\left(x_{k}\right), d_{k}\right)$ is checked for $0<\alpha_{k} \leq-\frac{1}{\left.2 M \| d_{k}\right) \|^{2}}\left(\nabla f\left(x_{k}\right), d_{k}\right)$

3. $f\left(x_{k}\right)$ is a convergent sequence

4. $\left(\nabla f\left(x_{k}\right), d_{k}\right) \longrightarrow 0$ when $k \longrightarrow+\infty$

5. The sequence $\left(x_{k}\right)_{k}$ converges to the $x^{*}$ solution of problem (1).

Proof.

1) $x_{0} \in X$, we suppose $x_{k} \in X$, we have $y_{k} \in X$ and $\left.\alpha_{k}=2^{-i} \alpha_{0} \in\right] 0.1\left[\right.$, then $x_{k+1}=x_{k}+\alpha_{k}\left(y_{k}-x_{k}\right) \in X$

2) By applying Taylor formula to $f$, we have:

$$
f\left(x_{k}+\alpha_{k} d_{k}\right)-f\left(x_{k}\right)=\alpha_{k}\left(\nabla f\left(x_{k}\right), d_{k}\right)+\frac{1}{2} \alpha_{k}^{2}\left(\nabla^{2} f\left(t_{k}\right) d_{k}, d_{k}\right)
$$

with $t_{k}=x_{k}+s \alpha_{k} d_{k}$ and $0<s<1$

Then, by using (3) we get :

$$
\left.f\left(x_{k}+\alpha_{k} d_{k}\right)-f\left(x_{k}\right) \leq \alpha_{k}\left(\nabla f\left(x_{k}\right), d_{k}\right)+\frac{1}{2} \alpha_{k}^{2} M \| d_{k}\right) \|^{2}
$$

So the condition:

$$
f\left(x_{k+1}\right)-f\left(x_{k}\right) \leq \frac{1}{2} \alpha_{k}\left(\nabla f\left(x_{k}\right), d_{k}\right)
$$

is checked for

$$
\left.\frac{1}{2} \alpha_{k}^{2} M \| d_{k}\right) \|^{2}+\alpha_{k}\left(\nabla f\left(x_{k}\right), d_{k}\right) \leq \frac{1}{2} \alpha_{k}\left(\nabla f\left(x_{k}\right), d_{k}\right)
$$

which gives that the condition is checked for

$$
\left.\frac{1}{2} \alpha_{k}^{2} M \| d_{k}\right) \|^{2} \leq-\frac{1}{2} \alpha_{k}\left(\nabla f\left(x_{k}\right), d_{k}\right)
$$

so $\alpha_{k}$ verifies

$$
\alpha_{k} \leq-\frac{1}{\left.M \| d_{k}\right) \|^{2}}\left(\nabla f\left(x_{k}\right), d_{k}\right)
$$

We can choose $\alpha_{k}=\alpha_{0} 2^{-i}$ with $i$ as the first clue that verifies:

It's always possible indeed

$$
2^{-i} \alpha_{0} \leq-\frac{1}{\left.M \mid \| d_{k}\right) \|^{2}}\left(\nabla f\left(x_{k}\right), d_{k}\right)
$$

$$
-\frac{1}{\left.M \mid d_{k}\right) \|^{2}}\left(\nabla f\left(x_{k}\right), d_{k}\right)>0
$$

and $2^{-n} \alpha_{0} \longrightarrow 0$ when $n \longrightarrow+\infty$.

3) We have:

$$
f\left(x_{k+1}\right)-f\left(x_{k}\right) \leq \frac{1}{2} \alpha_{k}\left(\nabla f\left(x_{k}\right), d_{k}\right) \leq 0
$$

then $\left(f\left(x_{k}\right)\right)$ is a declining sequence, so it converges. 
4) The condition on $\alpha_{k}$, gives:

$$
2^{-i+1} \alpha_{0}>-\frac{1}{\left.M \mid \| d_{k}\right) \|^{2}}\left(\nabla f\left(x_{k}\right), d_{k}\right)
$$

then

$$
2 \alpha_{k}>-\frac{1}{M\left\|d_{k}\right\|^{2}}\left(\nabla f\left(x_{k}\right), d_{k}\right)
$$

So

$$
\frac{1}{2} \alpha_{k}\left(\nabla f\left(x_{k}\right), d_{k}\right)<\frac{-1}{4 M\left\|d_{k}\right\|^{2}}\left(\nabla f\left(x_{k}\right), d_{k}\right)^{2}
$$

but

$$
f\left(x_{k+1}\right)-f\left(x_{k}\right) \leq \frac{1}{2} \alpha_{k}\left(\nabla f\left(x_{k}\right), d_{k}\right)
$$

then

$$
f\left(x_{k+1}\right)-f\left(x_{k}\right) \leq-\frac{1}{4 M\left\|d_{k}\right\|^{2}}\left(\nabla f\left(x_{k}\right), d_{k}\right)^{2}
$$

As a result:

$$
0 \leq\left(\nabla f\left(x_{k}\right), d_{k}\right)^{2} \leq 4 M\left\|d_{k}\right\|^{2}\left(f\left(x_{k}\right)-f\left(x_{k+1}\right)\right) \leq 4 M D^{2}\left(f\left(x_{k}\right)-f\left(x_{k+1}\right)\right)
$$

with $D=\max _{(x, y) \in X^{2}}\|x-y\|$, and by 3$)$ we have:

$$
\left(\nabla f\left(x_{k}\right), d_{k}\right) \underset{k \rightarrow+\infty}{\longrightarrow} 0 .
$$

5) By Taylor formula we have

$$
f\left(x^{*}\right)-f\left(x_{k}\right)=\left(\nabla f\left(x_{k}\right), x^{*}-x_{k}\right)+\frac{1}{2}\left(\nabla^{2} f\left(x_{k}+t_{k}\left(x^{*}-x_{k}\right)\right)\left(x^{*}-x_{k}\right), x^{*}-x_{k}\right)
$$

with $\left.t_{k} \in\right] 0,1[$. The relation (3) implies

$$
\frac{m}{2}\left\|x^{*}-x_{k}\right\|^{2}+\left(\nabla f\left(x_{k}\right), x^{*}-x_{k}\right) \leq f\left(x^{*}\right)-f\left(x_{k}\right) \leq 0
$$

then

$$
\frac{m}{2}\left\|x^{*}-x_{k}\right\|^{2} \leq\left(\nabla f\left(x_{k}\right), x_{k}-x^{*}\right)
$$

but

$$
\left(\nabla f\left(x_{k}\right), x_{k}-x^{*}\right)=\left(\nabla f\left(x_{k}\right), x_{k}-y_{k}\right)+\left(\nabla f\left(x_{k}\right), y_{k}-x^{*}\right)
$$

we also have by (2)

$$
\left(\nabla f\left(x_{k}\right), y_{k}-x^{*}\right) \leq 0
$$

then

$$
\frac{m}{2}\left\|x^{*}-x_{k}\right\|^{2} \leq\left(\nabla f\left(x_{k}\right), x_{k}-y_{k}\right)
$$

and

Using 4), we obtain

$$
\left\|x^{*}-x_{k}\right\|^{2} \leq-\frac{2}{m}\left(\nabla f\left(x_{k}\right), y_{k}-x_{k}\right)=-\frac{2}{m}\left(\nabla f\left(x_{k}\right), d_{k}\right) .
$$

$$
\left\|x^{*}-x_{k}\right\|^{2} \longrightarrow 0 \text { when } k \longrightarrow+\infty
$$




\section{Convergence Speed Assesment}

The demonstration of the theorem that gives the speed of convergence of the sequence $\mathrm{x}$ requires the following lemmas:

\section{Lemma 1}

We consider $x_{0} \in X$ and $E=\left\{x \in X\right.$ such that $\left.f(x) \leq f\left(x_{0}\right)\right\}$.

We suppose that there exists $\lambda>0$ and $R>0$ such that:

1. $f\left(\frac{x+y}{2}\right) \leq \frac{1}{2}(f(x)+f(y))-\lambda\|x-y\|^{2} \quad \forall x, \forall y \in X$

2. $\|\nabla f(x)\| \leq R \quad \forall x \in X$

Then:

$$
\frac{x_{k}+y_{k}}{2}+B\left(0, \frac{\lambda}{R}\left\|y_{k}-x_{k}\right\|^{2}\right) \subset E .
$$

Proof.

For $t \in B\left(0, \frac{\lambda}{R}\left\|y_{k}-x_{k}\right\|^{2}\right)$, applying the mean value theorem to $f$, we have:

$$
f\left(\frac{x_{k}+y_{k}}{2}+t\right)=f\left(\frac{x_{k}+y_{k}}{2}\right)+\left(\nabla f\left(t_{k}\right), t\right)
$$

with $t_{k}=\frac{x_{k}+y_{k}}{2}+s t$ and $0<s<1$,

then

$$
\begin{aligned}
f\left(\frac{x_{k}+y_{k}}{2}+t\right) & \leq \frac{1}{2}\left(f\left(x_{k}\right)+f\left(y_{k}\right)\right)-\lambda\left\|x_{k}-y_{k}\right\|^{2}+\left(\nabla f\left(t_{k}\right), t\right) \\
& \leq f\left(x_{0}\right)-\lambda\left\|x_{k}-y_{k}\right\|^{2}+\left\|\nabla f\left(t_{k}\right)\right\|\|t\| \\
& \leq f\left(x_{0}\right)-\lambda\left\|x_{k}-y_{k}\right\|^{2}+R\|t\|
\end{aligned}
$$

Using $t \in B\left(0, \frac{\lambda}{R}\left\|y_{k}-x_{k}\right\|^{2}\right)$ we obtain:

$$
f\left(\frac{x_{k}+y_{k}}{2}+t\right) \leq f\left(x_{0}\right)-\lambda\left\|x_{k}-y_{k}\right\|^{2}+R \frac{\lambda}{R}\left\|y_{k}-x_{k}\right\|^{2}=f\left(x_{0}\right)
$$

when therefore $\frac{x_{k}+y_{k}}{2}+t \in E$.

\section{Lemma 2}

Under the same assumptions as in Lemma 1, we have:

$$
\left(\nabla f\left(x_{k}\right), y_{k}-x_{k}\right) \leq-\frac{2 \lambda}{R}\left\|y_{k}-x_{k}\right\|^{2}\left\|\nabla f\left(x_{k}\right)\right\|
$$

Proof.

By (2), we have

$$
\left(\nabla f\left(x_{k}\right), y_{k}-x_{k}\right) \leq\left(\nabla f\left(x_{k}\right), y-x_{k}\right) \forall y \in E
$$

using Lemma 1

$$
\forall t \in B\left(0, \frac{\lambda}{R}\left\|y_{k}-x_{k}\right\|^{2}\right)
$$

we have:

$$
\left(\nabla f\left(x_{k}\right), y_{k}-x_{k}\right) \leq\left(\nabla f\left(x_{k}\right), \frac{x_{k}+y_{k}}{2}+t-x_{k}\right)=\left(\nabla f\left(x_{k}\right), \frac{y_{k}-x_{k}}{2}\right)+\left(\nabla f\left(x_{k}\right), t\right)
$$

So, for $t=-\frac{\lambda}{R\left\|\nabla f\left(x_{k}\right)\right\|}\left\|y_{k}-x_{k}\right\|^{2} \nabla f\left(x_{k}\right) \in B\left(0, \frac{\lambda}{R}\left\|y_{k}-x_{k}\right\|^{2}\right)$, we get :

$$
\left(\nabla f\left(x_{k}\right), y_{k}-x_{k}\right) \leq \frac{1}{2}\left(\nabla f\left(x_{k}\right), y_{k}-x_{k}\right)-\frac{\lambda}{R}\left\|y_{k}-x_{k}\right\|^{2}\left\|\nabla f\left(x_{k}\right)\right\|
$$

and then we have:

$$
\left(\nabla f\left(x_{k}\right), y_{k}-x_{k}\right) \leq-\frac{2 \lambda}{R}\left\|y_{k}-x_{k}\right\|^{2}\left\|\nabla f\left(x_{k}\right)\right\| .
$$

Theorem 2 Let $f$ be of class $C^{2}$ on $X$ convex bounded and suppose that there exists $m>0, M>0, \lambda>0, R>$ 0 , and $K>0$ such that: 
1. $m\|y\|^{2} \leq\left(\nabla^{2} f(x) y, y\right) \leq M\|y\|^{2} \quad \forall x, y \in X$

2. $f\left(\frac{x+y}{2}\right) \leq \frac{1}{2}(f(x)+f(y))-\lambda\|x-y\|^{2} \quad \forall x, \forall y \in X$

3. $K \leq\|\nabla f(x)\| \leq R \quad \forall x \in X$

Then :

1. $\left(f\left(x_{k}\right)-f\left(x^{*}\right)\right) \leq q^{k}\left(f\left(x_{0}\right)-f\left(x^{*}\right)\right)$

2. $\left\|x_{k}-x^{*}\right\|^{2} \leq L q^{k}$

with $q=\left(1-\frac{K}{2 M R}\right)$ and $L=2 \frac{f\left(x_{0}\right)-f\left(x^{*}\right)}{m}$.

Proof.

By (4), we have:

$$
2 \alpha_{k}>-\frac{1}{M\left\|d_{k}\right\|^{2}}\left(\nabla f\left(x_{k}\right), d_{k}\right)
$$

then

$$
\alpha_{k}>-\frac{1}{2 M\left\|d_{k}\right\|^{2}}\left(\nabla f\left(x_{k}\right), d_{k}\right)
$$

and

$$
\frac{1}{2} \alpha_{k}\left(\nabla f\left(x_{k}\right), d_{k}\right)<\frac{-1}{4 M\left\|d_{k}\right\|^{2}}\left(\nabla f\left(x_{k}\right), d_{k}\right)^{2}
$$

By the choice of $\alpha_{k}$, we have :

$$
f\left(x_{k+1}\right)-f\left(x_{k}\right) \leq \frac{1}{2} \alpha_{k}\left(\nabla f\left(x_{k}\right), d_{k}\right)
$$

then

$$
f\left(x_{k+1}\right)-f\left(x_{k}\right) \leq \frac{-1}{4 M\left\|d_{k}\right\|^{2}}\left(\nabla f\left(x_{k}\right), d_{k}\right)^{2}
$$

So

$$
\begin{gathered}
\left(f\left(x_{k+1}\right)-f\left(x^{*}\right)\right)-\left(f\left(x_{k}\right)-f\left(x^{*}\right)\right) \leq \frac{-1}{4 M\left\|d_{k}\right\|^{2}}\left(\nabla f\left(x_{k}\right), d_{k}\right)^{2} \\
\leq-\left(\nabla f\left(x_{k}\right), d_{k}\right)\left(\frac{1}{4 M\left\|d_{k}\right\|^{2}}\left(\nabla f\left(x_{k}\right), d_{k}\right)\right)
\end{gathered}
$$

By the lemma 2, we have

$$
\left(\nabla f\left(x_{k}\right), y_{k}-x_{k}\right) \leq-\frac{2 \lambda}{R}\left\|y_{k}-x_{k}\right\|^{2}\left\|\nabla f\left(x_{k}\right)\right\|
$$

then

$$
\left(\nabla f\left(x_{k}\right), y_{k}-x_{k}\right) \frac{1}{4 M\left\|d_{k}\right\|^{2}} \leq-\frac{\lambda}{2 M R}\left\|\nabla f\left(x_{k}\right)\right\|
$$

and

$$
-\left(\nabla f\left(x_{k}\right), d_{k}\right)\left(\frac{1}{4 M\left\|d_{k}\right\|^{2}}\left(\nabla f\left(x_{k}\right), d_{k}\right) \leq\left(\nabla f\left(x_{k}\right), d_{k}\right) \frac{\lambda}{2 M R}\left\|\nabla f\left(x_{k}\right)\right\|\right.
$$

from where

$$
\left(f\left(x_{k+1}\right)-f\left(x^{*}\right)\right)-\left(f\left(x_{k}\right)-f\left(x^{*}\right)\right) \leq \frac{\lambda}{2 R M}\left\|\nabla f\left(x_{k}\right)\right\|\left(\nabla f\left(x_{k}\right), y_{k}-x_{k}\right)
$$

By Taylor formula and (3) we have:

$$
\frac{m}{2}\left\|x_{k}-x^{*}\right\|^{2}+\left(\nabla f\left(x_{k}\right), x^{*}-x_{k}\right) \leq f\left(x^{*}\right)-f\left(x_{k}\right)
$$

then

$$
\left(\nabla f\left(x_{k}\right), x^{*}-x_{k}\right) \leq f\left(x^{*}\right)-f\left(x_{k}\right)
$$

By (2), we also have

$$
\left(\nabla f\left(x_{k}\right), y_{k}-x_{k}\right) \leq\left(\nabla f\left(x_{k}\right), x^{*}-x_{k}\right)
$$

therefore 


$$
\left(f\left(x_{k+1}\right)-f\left(x^{*}\right)\right)-\left(f\left(x_{k}\right)-f\left(x^{*}\right)\right) \leq \frac{\lambda}{2 M R}\left\|\nabla f\left(x_{k}\right)\right\|\left(f\left(x^{*}\right)-f\left(x_{k}\right)\right)
$$

We deduce thus:

$$
\left(f\left(x_{k+1}\right)-f\left(x^{*}\right)\right)-\left(f\left(x_{k}\right)-f\left(x^{*}\right)\right) \leq \frac{\lambda}{2 R M} K\left(f\left(x^{*}\right)-f\left(x_{k}\right)\right)
$$

from where

$$
\left(f\left(x_{k+1}\right)-f\left(x^{*}\right)\right) \leq\left(1-\frac{\lambda K}{2 M R}\right)\left(f\left(x_{k}\right)-f\left(x^{*}\right)\right)
$$

Thus, we deduce

$$
\left(f\left(x_{k}\right)-f\left(x^{*}\right)\right) \leq\left(1-\frac{\lambda K}{2 M R}\right)^{k}\left(f\left(x_{0}\right)-f\left(x^{*}\right)\right)
$$

2) By applying Taylor's theorem and (3), we have:

$$
\frac{m}{2}\left\|x_{k}-x^{*}\right\|^{2}+\left(\nabla f\left(x^{*}\right), x_{k}-x^{*}\right) \leq f\left(x_{k}\right)-f\left(x^{*}\right)
$$

Using (1), we have:

$$
\left(\nabla f\left(x^{*}\right), x_{k}-x^{*}\right) \geq 0
$$

then

$$
\frac{m}{2}\left\|x_{k}-x^{*}\right\|^{2} \leq f\left(x_{k}\right)-f\left(x^{*}\right)
$$

we deduce

$$
\left\|x_{k}-x^{*}\right\|^{2} \leq \frac{2}{m}\left(1-\frac{\lambda K}{2 M R} K\right)^{k}\left(f\left(x_{0}\right)-f\left(x^{*}\right)\right)
$$

we finally obtain : $\left\|x_{k}-x^{*}\right\|^{2} \leq L q^{k}$

with $q=\left(1-\frac{\lambda K}{2 M R}\right)$ and $L=2 \frac{f\left(x_{0}\right)-f\left(x^{*}\right)}{m}$.

\section{Numerical Experiments}

In this section, we present some numerical experiments. We compare the new method N.M. with a quadratic programming method Q.M. This comparison is summarized in the tables which give the number of iterations, the associated residual norms for each method and the convergence time.

\section{Example 1:}

We consider the following problem: $\left\{\begin{array}{l}\text { Minimize } 0.5 x_{1}^{2}+0.5 x_{2}^{2}-2 x_{1} x_{2}-x_{1}-2 x_{2} \\ \text { subject to : }\left\{\begin{array}{l}x_{1}+x_{2} \leq 1 \\ x_{1} \geq 0, \text { and } x_{2} \geq 0 .\end{array}\right.\end{array}\right.$

where $x_{0}=(0.2,0.8)$ 
Table 1. Numerical result of example 1

\begin{tabular}{c|c|c}
\hline Iterations & N.M. & Q.M. \\
\hline 1 & 0.035555555555556 & 0.035555 \\
\hline 2 & 0.008888888878264 & $3.000000 \mathrm{e}+00$ \\
\hline 3 & 0.002222221994011 & $1.500000 \mathrm{e}-03$ \\
\hline 4 & $5.555551107565639 \mathrm{e}-04$ & $5.379119 \mathrm{e}-06$ \\
\hline 5 & $1.388888222550025 \mathrm{e}-04$ & $1.159073 \mathrm{e}-12$ \\
\hline 6 & $3.472221628893632 \mathrm{e}-05$ & \\
\hline 7 & $8.680564444023085 \mathrm{e}-06$ & \\
\hline 8 & $2.170143031633367 \mathrm{e}-06$ & \\
\hline 9 & $1.356348304996638 \mathrm{e}-07$ & \\
\hline 10 & $3.390935719608362 \mathrm{e}-08$ & \\
\hline 11 & $8.477501597801585 \mathrm{e}-09$ & \\
\hline 12 & $2.119537653982382 \mathrm{e}-09$ & \\
\hline 13 & $5.299249731595960 \mathrm{e}-10$ & \\
\hline 14 & $1.325218040089173 \mathrm{e}-10$ & \\
\hline 15 & $3.314058366479270 \mathrm{e}-11$ & \\
\hline 16 & $8.295162550844132 \mathrm{e}-12$ & \\
\hline CPU & $0.141647 \mathrm{~s}$ & 1.584391 \\
\hline
\end{tabular}

$$
x^{*}=(0.333333333463281,0.666666666355120)
$$

\section{Example 2:}

We consider the following problem:

$\left\{\begin{array}{l}\text { Minimize } x_{1}^{2}+x_{2}^{2}+x_{3}^{2}+x_{4}^{2}-2 x_{1}-x_{2}-3 x_{4} \\ \text { subject to: }\left\{\begin{array}{l}2 x_{1}+x_{2}+x_{3}+4 x_{4} \leq 7 \\ x_{1}+x_{2}+2 x_{3}+x_{4} \leq 6 \\ x_{1} \geq 0, x_{2} \geq 0, x_{3} \geq 0\end{array} \text { and } x_{4} \geq 0 .\right.\end{array}\right.$

where $x_{0}=(1 ; 1 ; 0 ; 1)$.

Table 2. Numerical result of example 2

\begin{tabular}{c|c|c}
\hline Iterations & N.M. & Q.M. \\
\hline 1 & 0.392827780807241 & 0.392827 \\
\hline 2 & 0.140918977309031 & $3.370370 \mathrm{e}+00$ \\
\hline 3 & 0.023263204565265 & $3.809960 \mathrm{e}-01$ \\
\hline 4 & 0.005240614092247 & $1.904980 \mathrm{e}-04$ \\
\hline 5 & 0.002297400115120 & $6.923784 \mathrm{e}-07$ \\
\hline 6 & $5.220697158989774 \mathrm{e}-04$ & $3.461880 \mathrm{e}-10$ \\
\hline 7 & $8.492267636464691 \mathrm{e}-05$ & $1.740830 \mathrm{e}-13$ \\
\hline 8 & $2.220525070921954 \mathrm{e}-05$ & \\
\hline 849 & $9.298572946438025 \mathrm{e}-06$ & \\
\hline 10 & $1.703274996615137 \mathrm{e}-06$ & \\
\hline 11 & $3.159987804839340 \mathrm{e}-07$ & \\
\hline 12 & $2.305206106734471 \mathrm{e}-07$ & \\
\hline 13 & $4.409278531393869 \mathrm{e}-08$ & \\
\hline 14 & $1.538823542774516 \mathrm{e}-08$ & \\
\hline 15 & $5.064006381347326 \mathrm{e}-09$ & \\
\hline 16 & $1.336291322194917 \mathrm{e}-09$ & \\
\hline 17 & $6.632606629537780 \mathrm{e}-10$ & \\
\hline CPU & $0.268475 \mathrm{~s}$ & $0.689212 \mathrm{~s}$ \\
\hline
\end{tabular}




\section{Example 3:}

We consider the following problem:

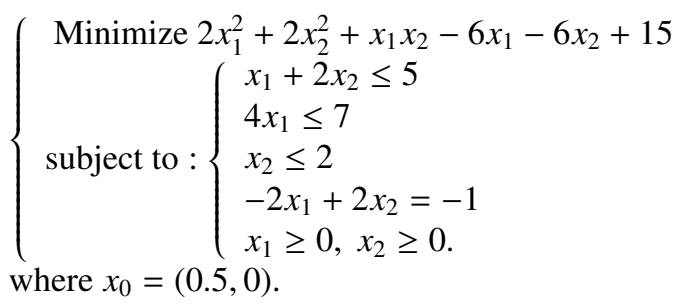

Table 3. Numerical result of example 3

\begin{tabular}{c|c|c}
\hline Iterations & N.M. & Q.M. \\
\hline 1 & 1.804999935863038 & 1.804 \\
\hline 2 & 0.488071966640245 & $2.351 \mathrm{e}+00$ \\
\hline 3 & 0.131974651411347 & $3.267 \mathrm{e}-02$ \\
\hline 4 & 0.035685941396897 & $9.112 \mathrm{e}-04$ \\
\hline 5 & 0.009649476122220 & $5.051 \mathrm{e}-07$ \\
\hline 6 & 0.002609217148293 & $2.526 \mathrm{e}-10$ \\
\hline 7 & $7.055317223436338 \mathrm{e}-04$ & \\
\hline 8 & $1.907754546998011 \mathrm{e}-04$ & \\
\hline 9 & $5.158548814694946 \mathrm{e}-05$ & \\
\hline 10 & $1.394863229703878 \mathrm{e}-05$ & \\
\hline 11 & $3.771666855020771 \mathrm{e}-06$ & \\
\hline 12 & $1.019836139137253 \mathrm{e}-06$ & \\
\hline 13 & $2.757520022330317 \mathrm{e}-07$ & \\
\hline 14 & $7.455724534172043 \mathrm{e}-08$ & \\
\hline 15 & $2.015711684713288 \mathrm{e}-08$ & \\
\hline 16 & $5.448966664930066 \mathrm{e}-09$ & \\
\hline 17 & $6.632606629537780 \mathrm{e}-10$ & \\
\hline 18 & $1.472544491803056 \mathrm{e}-09$ & \\
\hline 19 & $3.977165539178516 \mathrm{e}-10$ & \\
\hline 20 & $1.072955802546778 \mathrm{e}-10$ & \\
\hline 21 & $2.889284321440711 \mathrm{e}-11$ & \\
\hline 22 & $7.759896562204401 \mathrm{e}-12$ & \\
\hline CPU & $0.607344 \mathrm{~s}$ & $0.689212 \mathrm{~s}$ \\
\hline & &
\end{tabular}

$$
x^{*}=(1.449999999545066,0.949999999545066)
$$

\section{Conclusions}

1. The method is simple to describe.

2. The method allows to solve a large class of optimization problems with constraints:

$$
\left\{\begin{array}{l}
\operatorname{Minf}(x) \\
A x \leq b \\
x \geq 0
\end{array}\right.
$$

where $A$ the matrix of constraint coefficients, $x$ a variable vector, $b$ the vector of variables and 0 a vector made up only of zeros.

At each iteration $x_{k}$ the direction $d_{k}=y_{k}-x_{k}$ is determined by solving the following problem:

$$
\left\{\begin{array}{l}
\operatorname{Min}\left(c_{k}, y\right)=\left(c_{k}, y_{k}\right) \\
\operatorname{Ay} \leq b \\
y \geq 0
\end{array}\right.
$$


where $c_{k}=\nabla f\left(x_{k}\right)$ will be the vector of coefficients of the objective function.

3. At each step, only the objective function is changed. The constraints remain the same.

\section{References}

Dennis, J. E., \& Schnabel, R. B. (1996). Numerical methods for unconstrained optimization and nonlinear equations. Philadelphia: Soc. for Industrial and Applied Mathematics. https://doi.org/10.1137/1.9781611971200

Moulin, H., \& Fougelman-Souli, F. (1979). Convexité dans les mathématiques de la décision. Hermann, Paris.

Rondepierre, A. (2017). Méthodes numériques pour l'optimisation non linéaire déterministe.

Ortega, J. M., \& Rheinboldt, W. C. (2000). Iterative solution of nonlinear equations in several variables. Philadelphia (Pa.): Society for industrial and applied mathematics. https://doi.org/10.1016/C2013-0-11263-9

Rhanizar, B. (2002). Nonlinear Multiple Hybrid Procedures For Solving Some Constrained Nonlinear Optimization problems. Applicationes mathematicae, 29(2), 185-198. http://dx.doi.org/10.4064/am29-2-5

\section{Copyrights}

Copyright for this article is retained by the author(s), with first publication rights granted to the journal.

This is an open-access article distributed under the terms and conditions of the Creative Commons Attribution license (http://creativecommons.org/licenses/by/4.0/). 